\title{
Multiplicity mod 2 as a Metric Invariant
}

\author{
Guillaume Valette
}

Received: 14 August 2008 / Revised: 9 June 2009 / Accepted: 9 June 2009 /

Published online: 23 June 2009

(C) Springer Science+Business Media, LLC 2009

\begin{abstract}
We study the multiplicity modulo 2 of real analytic hypersurfaces. We prove that, under some assumptions on the singularity, the multiplicity modulo 2 is preserved by subanalytic bi-Lipschitz homeomorphisms of $\mathbb{R}^{n}$. In the first part of the paper, we find a subset of the tangent cone which determines the multiplicity mod 2 and prove that this subset of $S^{n}$ is preserved by the antipodal map. The study of such subsets of $S^{n}$ enables us to deduce the subanalytic metric invariance of the multiplicity modulo 2 under some extra assumptions on the tangent cone. We also prove a real version of a theorem of Comte, and yield that the multiplicity modulo 2 is preserved by arc-analytic bi-Lipschitz homeomorphisms.
\end{abstract}

Keywords Real analytic hypersurfaces $\cdot$ Multiplicity $\cdot$ Metric invariant

\section{Introduction}

During the three last decades, various criteria for topological stability of singular algebraic or analytic subsets were given. Numerous topological invariants were also found, especially in algebraic geometry. For classification problems, invariants are very useful to distinguish different classes. More recently, attention started to focus on the metric type of singularities. But if we have lots of invariants describing the topological type of singularities, we dispose of rather a few devoted to the metric type

This paper was written during postdoctoral stage at University of Toronto, and is partially supported by the Polish KBN grant 0235/P03/2004/27.

G. Valette $(\bowtie)$

Department of Mathematics, University of Toronto, 40 St. George st, Toronto, ON M5S 2E4, Canada e-mail: gvalette@math.toronto.edu

Present address:

G. Valette

Instytut Matematyczny PAN, ul. Św. Tomasza 30, 31-027 Kraków, Poland 
of a singularity and very few of them are explicitly computable from the equation of an analytic set.

This paper deals with analytic hypersurfaces of $\mathbb{R}^{n}$ and focuses on their multiplicity. By multiplicity of a real analytic germ of a hypersurface defined by a reduced analytic equation $P=0$, we mean the lowest degree in the expansion of $P$. In complex algebraic geometry the multiplicity has a geometric interpretation as the cardinality of a generic fiber of a generic projection onto a hyperplane. If we consider the multiplicity modulo 2 , this geometric interpretation also holds for real analytic hypersurfaces.

O. Zariski asked whether two germs of analytic hypersurfaces of $\mathbb{C}^{n}$ which are homeomorphic have the same multiplicity. The Zariski conjecture is still open more than thirty years later. It is easy to find examples of real analytic sets which are homeomorphic and which have different multiplicities, even modulo 2 . For instance, a cusp defined by $\left\{(x ; y) \in \mathbb{R}^{2} / y^{2}=x^{3}\right\}$ is homeomorphic to a straight line. This raises a very natural question: is the multiplicity $\bmod 2$ a metric invariant for real analytic hypersurfaces?

Metric classification refers to classification of bi-Lipschitz maps $h: \mathbb{R}^{n} \rightarrow \mathbb{R}^{n}$, which are the maps satisfying:

$$
\frac{1}{C}\left|x-x^{\prime}\right| \leq\left|h(x)-h\left(x^{\prime}\right)\right| \leq C\left|x-x^{\prime}\right|,
$$

for some constant $C \geq 1$. The smallest constant $C$ for which this inequality holds is then called the Lipschitz constant of $h$ and is denoted by $\operatorname{Lip}(h)$.

One will observe that in the above example the two given curves are homeomorphic but not bi-Lipschitz homeomorphic.

In this paper we investigate this problem and give a partial answer. We prove that the multiplicity modulo 2 of real analytic hypersurfaces is preserved by subanalytic bi-Lipschitz maps under an extra assumption on the singularity which, roughly speaking, is somewhat stronger than requiring that the singularities of the link be isolated. Namely, we introduce what we call the odd part of the tangent cone and are able to prove invariance of the multiplicity modulo 2 in the case where this subset has only isolated singularities (Theorem 5.2). We prove this result for all the hypersurfaces of $\mathbb{R}^{3}$ as well (Theorem 5.1). We also prove a real version of a theorem of George Comte: the multiplicity modulo 2 of a real analytic hypersurface is preserved by a subanalytic bi-Lipschitz homeomorphism whose Lipschitz constant is sufficiently close to one (Theorem 5.4). For complex analytic sets, a similar theorem has been proved by Comte in [2]. Subanalytic bi-Lipschitz equivalence is a good equivalence relation since it has been proved in [9] that it does not admit continuous moduli.

It is proved in [3] that any semialgebraic subset of $S^{n}$ is the tangent cone of an algebraic singular set. It would be interesting to investigate the question of whether every semialgebraic subset (or subanalytic subset) of $S^{n}$ which is preserved by the antipodal map may be the odd part of the tangent cone of a subanalytic singular set.

Content of the Paper We start by showing (Proposition 2.4) how the multiplicity mod 2 can be computed from a part of the tangent cone (called the odd part of the tangent cone). In Sect. 2 we prove that the odd part of the tangent cone is equivariant 
(preserved by the antipodal map). Section 3 is devoted to the topology of equivariant submanifolds of $S^{n}$. This enables us to show that the multiplicity modulo 2 is preserved by arc-analytic bi-Lipschitz homeomorphisms. Relating the multiplicity to the Euler characteristic of the odd part of the tangent cone when it has only isolated singularities, we prove that the multiplicity modulo 2 is a topological invariant of the odd part of the tangent cone. The last section concludes with our equimultiplicity results, which are the main results of this article.

Notation Throughout this paper we write $a \equiv b$ if $a$ and $b$ are two integers which are equal $\bmod 2$. We write $a \equiv b[4]$ if they are equal modulo 4 . Given a subset $X$ of $\mathbb{R}^{n}$ we denote by $\operatorname{cl}(X)$ its closure and $|$.$| stands for the Euclidean norm of \mathbb{R}^{n}$. We write $\mathbb{R}_{>0}$ for the positive real numbers.

All the stratifications are assumed to be topologically trivial. Given a subanalytic set $C$ we write $\operatorname{Sing} C$ for the singular locus of $C$, i.e. the set of all the points of $C$ at which the set $C$ is not a topological manifold.

Given $\lambda \in S^{n}$ we write $\pi_{\lambda}: \mathbb{R}^{n+1} \rightarrow \mathbb{R}^{n+1}$ for the orthogonal projection onto $\lambda^{\perp}$.

\section{Euler Cycles}

In this section we relate the multiplicity of analytic hypersurfaces to their tangent cones (Proposition 2.4).

Notation The projection $\pi_{\lambda}$ defined above induces a mapping $\pi_{\lambda}^{\prime}: S^{n} \backslash \pm \lambda \rightarrow S^{n-1}$ defined by $\pi_{\lambda}^{\prime}(u):=\frac{\pi_{\lambda}(u)}{\left|\pi_{\lambda}(u)\right|}$. Let $C$ be a subanalytic subset of $S^{n}$. We set

$$
\varphi_{\lambda}(x):=\operatorname{card} \pi_{\lambda}^{\prime-1}(x) \cap C
$$

(which is finite for generic $x$ and $\lambda$ ).

We start by characterizing the sets for which $\varphi_{\lambda}$ is constant generically modulo 2 .

Definition 2.1 An (n-1)-dimensional subanalytic set $C$ is said to be an Euler cycle if it is a closed set and if, for a stratification of $C$ (and hence for any), the number of $(n-1)$-dimensional strata containing a given $(n-2)$-dimensional stratum in their closure is even.

This is a well-known property of real algebraic sets (see for instance [1], Theorem 11.1.1).

Proposition 2.2 Let $C$ be a subanalytic subset of $S^{n}$ and let $\lambda \in S^{n}$ be generic. Then, $\varphi_{\lambda}(x)$ is constant modulo 2 for $x$ generic in $S^{n-1}$ if and only if $C$ is an Euler cycle.

Proof Considering a cylindrical decomposition we may extract a finite partition of $S^{n-1}$ such that $\varphi_{\lambda}$ is constant on each element of this partition. Fix some stratifications of $C$ and $S^{n-1}$ respectively in such a way that $\pi_{\lambda}^{\prime}$ maps submersively strata onto strata. Choose a $(n-2)$-dimensional stratum $\beta \subset S^{n-1}$. Then there exist exactly two strata of $S^{n-1}$, say $\alpha$ and $\alpha^{\prime}$, containing $\beta$ in their closure.

Let $\pi_{\lambda}^{\prime-1}(\beta) \cap C:=\left\{\beta_{1}, \ldots, \beta_{k}\right\}, \pi_{\lambda}^{\prime-1}(\alpha) \cap C:=\left\{\alpha_{1}, \ldots, \alpha_{m}\right\}$ and $\pi_{\lambda}^{\prime-1}\left(\alpha^{\prime}\right) \cap$ $C:=\left\{\alpha_{1}^{\prime}, \ldots, \alpha_{m^{\prime}}^{\prime}\right\}$. 
For $i \leq k$ we set

$$
j_{i}:=\operatorname{card}\left\{\alpha_{l} / \beta_{i} \subset \operatorname{cl}\left(\alpha_{l}\right)\right\}
$$

(resp. $\left.j_{i}^{\prime}=\operatorname{card}\left\{\alpha_{l}^{\prime} / \beta_{i} \subset \operatorname{cl}\left(\alpha_{l}^{\prime}\right)\right\}\right)$.

If $C$ is an Euler cycle then $j_{i} \equiv j_{i}^{\prime}$ for each $i$. So $\sum_{i=1}^{k} j_{i} \equiv \sum_{i=1}^{k} j_{i}^{\prime}$ and hence $m \equiv m^{\prime}$.

To prove the converse, assume that $C$ is not an Euler cycle. Thus, the condition of the definition fails, say at $\beta_{1}$. Let $\beta=\pi_{\lambda}^{\prime}\left(\beta_{1}\right)$, then $\beta$ lies in the closure of two $(n-1)$ dimensional strata of $S^{n-1}$, say $\alpha$ and $\alpha^{\prime}$. But, as we assumed that the condition of Definition 2.1 fails along the stratum $\beta_{1}$, this implies (with the notation introduced in (2.1)): $j_{1} \equiv 1+j_{1}^{\prime}$.

For a sufficiently generic $\lambda$ we may assume that $\pi_{\lambda}^{\prime-1}\left(\pi_{\lambda}^{\prime}\left(\beta_{1}\right) \cap C\right)$ is the union of $\beta_{1}$ and of some strata lying in the closure of exactly two strata (this holds in particular if they lie in the regular locus of $C$ ). This implies $j_{i} \equiv j_{i}^{\prime}$ for $i>1$ and hence $m \equiv 1+m^{\prime}$.

Hence, for an Euler cycle $C$, we denote by $m_{\lambda}(C)$ the generic value modulo 2 of $\varphi_{\lambda}(x)$. A priori this depends on $\lambda$.

\subsection{Multiplicity $\bmod 2$ and Tangent Cone}

If $A$ is the germ of an analytic hypersurface of $\mathbb{R}^{n+1}$ it is well known that $\operatorname{card} \pi_{\lambda}^{-1}(x) \cap A$ is constant modulo 2 for $\lambda$ generic in $S^{n}$ and $x$ generic in $\mathbb{R}^{n}$ sufficiently close to zero. This number coincides with the multiplicity $\bmod 2$ of $A$ at the origin; we will denote it by $m_{A}$.

We are going to relate the notion of multiplicity $\bmod 2$ of a real analytic set to the tangent cone. We recall that the tangent cone of a subanalytic set $A$ of $\mathbb{R}^{n+1}$ is defined by

$$
C_{A}=\left\{\lambda \in S^{n} / \exists \gamma:[0 ; \varepsilon[\rightarrow A, \gamma(t)=\lambda t+\cdots\} .\right.
$$

Usually, the tangent cone is rather the cone over this subset, but it will be more convenient to work with subsets of $S^{n}$.

Every subanalytic map germ $h:\left(\mathbb{R}^{n+1} ; 0\right) \rightarrow\left(\mathbb{R}^{n+1} ; 0\right)$ is Lipschitz differentiable in the sense that $\lim _{r \rightarrow 0} \frac{h(r x)}{r}$ exists, for any $x \in \mathbb{R}^{n}$. Indeed, the arc $r \mapsto h(r x)$ is mapped onto a subanalytic arc which must have an endpoint. Denote by $d_{0} h(x)$ the latter limit. Given a subanalytic bi-Lipschitz homeomorphism $h:\left(\mathbb{R}^{n+1} ; 0\right) \rightarrow$ $\left(\mathbb{R}^{n+1} ; 0\right)$ we may now define a mapping $h^{\prime}: S^{n} \rightarrow S^{n}$ by setting

$$
h^{\prime}(x):=\frac{d_{0} h(x)}{\left|d_{0} h(x)\right|} .
$$

The bi-Lipschitz condition implies that $d_{0} h(x)$ is nonzero so that $h^{\prime}$ is well defined.

We will deal with arc-analytic functions. They have been introduced by Kurdyka in [7]. We recall their definition.

Definition 2.3 Let $U$ be an open subset of the origin in $\mathbb{R}^{n+1}$. A function $f: U \rightarrow \mathbb{R}$ is arc-analytic if for every analytic arc $\gamma:[0 ; \varepsilon) \rightarrow U$ the function $f \circ \gamma$ is analytic. 
It is possible to compute the multiplicity $\bmod 2$ of an Euler cycle from its tangent cone.

Proposition 2.4 Let $A$ be a germ (at 0 ) of an analytic hypersurface of $\mathbb{R}^{n+1}$. Then, there exists an Euler cycle $C_{A}^{\prime} \subset C_{A}$ of pure dimension $(n-1)$, if nonempty, such that

$$
m_{\lambda}\left(C_{A}^{\prime}\right) \equiv m_{A}
$$

for $\lambda$ generic.

Proof First we are going to associate, to a given hypersurface $A$, a subanalytic function $s_{A}: C_{A} \backslash N \rightarrow \mathbb{N}$, where $N$ is a subanalytic subset of $S^{n}$ with $\operatorname{dim} N \leq n-2$, such that for any generic $\lambda \in S^{n} \backslash C_{A}$ we have

$$
\sum_{y \in \pi_{\lambda}^{\prime}-1} s_{A}(y)=\operatorname{card} \pi_{\lambda}^{-1}(r x) \cap A
$$

for any generic $x \in S^{n-1}$ and any positive real number $r$ small enough.

A subanalytic function which takes only integral values is constant on the strata of a subanalytic stratification. Thus, it will be enough to define $C_{A}^{\prime}$ as the closure of the union of the strata on which $s_{A}$ is odd, in order to get a subset having the desired property.

To define the function $s_{A}$ let us consider the spherical blowing-up at 0 :

$$
e: S^{n} \times \mathbb{R}^{+} \rightarrow \mathbb{R}^{n+1}
$$

defined by $e(x ; r)=r x$. Then note that

$$
C_{A}=\operatorname{cl}\left(e^{-1}(A)\right) \cap S^{n} \times\{0\},
$$

and take a stratification $S$ of $c l\left(e^{-1}(A)\right)$ compatible with $C_{A}$. We may construct simultaneously a stratification $S^{\prime}$ of $\mathbb{R}^{n}$ in such a way that $\pi_{\lambda} \circ e$ maps submersively strata onto strata. Now, for $y$ in an $(n-1)$-dimensional stratum $Y$ of $S$ included in $C_{A}$, we can define $s_{A}(y)$ as the number of strata $Y^{\prime}$ satisfying $\operatorname{cl}\left(Y^{\prime}\right) \backslash Y^{\prime} \supseteq Y$.

Let $y \in S^{n}$ be generic. For $r$ small enough we set

$$
u:=\operatorname{card} \pi_{\lambda}^{-1}(r y) \cap A .
$$

Let also:

$$
\pi_{\lambda}^{-1}(e(y ; r)) \cap A:=\left\{\gamma_{1}(r), \ldots, \gamma_{u}(r)\right\}
$$

and $\pi_{\lambda}^{\prime-1}(y) \cap C_{A}:=\left\{y_{1}, \ldots, y_{p}\right\}$.

Note that each path $\tilde{\gamma}_{i}:=e^{-1}\left(\gamma_{i}\right)$ ends at some $y_{i}$. Hence, to check (2.3) it is enough to check that the number of $\tilde{\gamma}_{j}$ 's ending at $y_{i}$ is precisely $s_{A}\left(y_{i}\right)$. Fix $j \leq p$ and let $Y_{1}, \ldots, Y_{s_{A}\left(y_{j}\right)}$ be the strata such that $y_{j} \in \operatorname{cl}\left(Y_{i}\right), 1 \leq i \leq s_{A}\left(y_{j}\right)$. As the map $\pi_{\lambda} \circ e_{\mid Y_{i}}$ is proper and submersive, it is a homeomorphism (we may assume the strata homeomorphic to open balls of $\mathbb{R}^{n}$ ). Therefore, the $\gamma_{k}$ 's may only belong to 
distinct $Y_{i}$. It means that $\sum s_{A}\left(y_{j}\right) \leq u$. Hence, it is enough to check that each $Y_{i}$ contains one of the $\gamma_{k}$ 's.

Fix $i \leq m$. By the curve selection lemma we can find a curve $\tilde{\gamma}$ in $Y_{i}$ ending at $y_{j}$. Hence, we see that $\gamma=e(\tilde{\gamma})$ is a subanalytic curve ending at the origin and tangent to $y_{j}$. Let us remark that the vector $y$ is tangent to $\pi_{\lambda}(\gamma)$. Let $M$ be the stratum containing $e(y ; r)$. Note that $\pi_{\lambda} \circ e: Y_{i} \rightarrow M$ is a homeomorphism. As $y$ is generic we can assume that $\operatorname{dim} M=n$ and that $y$ is not in the tangent cone of the boundary of $M$. But as the vector $y$ is tangent to $\pi_{\lambda}(\gamma(r))$ this implies that $e(y ; r)$ is in the image of $e \circ \pi_{\lambda \mid Y_{i}}$ for $r$ small enough, as required.

By definition, for $x$ generic, $s_{A}(x)$ is nothing but the number of connected components of

$$
e^{-1}(A \backslash\{0\}) \cap B(x ; \varepsilon),
$$

for $\varepsilon>0$ small enough. It is thus independent of the considered stratification. As $C_{A}^{\prime}$ is defined as the closure of the set of points in $C_{A}^{\prime}$ at which $s_{A}$ is odd, we see that $C_{A}^{\prime}$ is independent of the stratification.

Note that the fact that $C_{A}^{\prime}$ is an Euler cycle follows from the preceding proposition.

Proposition 2.5 (Metric invariance of $C_{A}^{\prime}$ ) Given two germs of analytic hypersurfaces $A$ and $B$ and a germ of subanalytic or arc-analytic bi-Lipschitz homeomorphism $h:\left(\mathbb{R}^{n+1} ; 0\right) \rightarrow\left(\mathbb{R}^{n+1} ; 0\right)$ such that $h(A)=B$, we have

$$
h^{\prime}\left(C_{A}^{\prime}\right)=C_{B}^{\prime}
$$

(see (2.2) for $\left.h^{\prime}\right)$.

Proof The map $h^{\prime}$ sends $C_{A}$ into $C_{B}$. Two rays $r x$ and $r x^{\prime}$ are mapped onto subanalytic arcs which may not be tangent since $h$ is bi-Lipschitz. Hence, $h^{\prime}$ is a homeomorphism. Let $s_{A}$ be the function defined in the proof of the previous proposition for $A$ and denote by $s_{B}$ the analogous function for $B$.

Recall that, for $x$ generic, $s_{A}(x)$ is the number of connected components of

$$
\left(\operatorname{cl}\left(e^{-1}(A)\right) \backslash C_{A}\right) \cap B(x ; \epsilon)
$$

(for $\epsilon$ small enough), where $e: S^{n} \times \mathbb{R}^{+} \rightarrow \mathbb{R}^{n+1}$ is the spherical blowing-up $e(x ; r)=r x$. As $h$ is a homeomorphism, we clearly have $s_{B}\left(h^{\prime}(x)\right)=s_{A}(x)$, for any $x$ generic in $C_{A}$, and hence $h^{\prime}$ sends $C_{A}^{\prime}$ onto $C_{B}^{\prime}$.

The function $s_{A}$ in the proof corresponds to the multiplicities introduced by K. Kurdyka and G. Raby in [8]. In the latter paper, the authors point out that those multiplicities are analytic invariants. The above argument proves that they are actually subanalytic metric invariants.

As the set $C_{A}^{\prime}$ corresponds to the points having an odd multiplicity we will call it the odd part of the tangent cone. This section shows that only the odd part is relevant to determine the multiplicity $\bmod 2$ of a given analytic hypersurface.

We shall need the following lemma. 
Lemma 2.6 Let $h:\left(\mathbb{R}^{n+1} ; 0\right) \rightarrow\left(\mathbb{R}^{n+1} ; 0\right)$ be a germ of a subanalytic bi-Lipschitz homeomorphism with Lipschitz constant L. Then $h^{\prime}$ satisfies, for any $x$ and $x^{\prime}$ in $S^{n}$,

$$
\frac{1}{L^{2}}\left|x-x^{\prime}\right|-\delta \leq\left|h^{\prime}(x)-h^{\prime}\left(x^{\prime}\right)\right| \leq L^{2}\left|x-x^{\prime}\right|+L \delta,
$$

where $\delta:=L-1$.

Proof Define

$$
\tilde{h}_{r}(x):=\left(|h(r x)| ; \frac{h(r x)}{|h(r x)|}\right) .
$$

Now observe that, due to the bi-Lipschitz character of $h$, we must have

$$
|| h(r x)|-r| \leq \delta r .
$$

Therefore, from the definition of $\tilde{h}_{r}$, an easy computation shows that

$$
\left|\tilde{h}_{r}(x)-\tilde{h}_{r}\left(x^{\prime}\right)\right| \leq L^{2}\left|x-x^{\prime}\right|+L \delta
$$

Arguing on $\tilde{h}_{r}^{-1}$ we get the other inequality.

\section{Hypersurfaces and Paths}

In this section the letter $C$ will stand for a subanalytic closed Euler cycle of $S^{n}, n>1$.

Definition 3.1 A subanalytic $C^{1}$ arc $\gamma:[0 ; 1] \rightarrow S^{n}$ is said to be an allowed path for $C$ if for every $t$ in the set $I_{\gamma}=\{t \in[0 ; 1] / \gamma(t) \in C\}$ the point $\gamma(t)$ is a regular point of $C$ at which the mapping $\gamma$ is transverse to $C$.

Note that the set $I_{\gamma}$ is always finite, so that we may define the length of an allowed path $\gamma$ by

$$
\lg _{C} \gamma=\operatorname{card} I_{\gamma}
$$

It is easy to see that two points of the complement of $C$ in $S^{n}$ may be joint by an allowed path. Therefore, we may define, for $\lambda$ and $\mu$ in $S^{n} \backslash C$,

$$
d_{C}(\lambda ; \mu):=\min \left\{\lg _{C} \gamma / \gamma \text { allowed path joining } \lambda \text { and } \mu\right\} .
$$

This is not a distance on $S^{n} \backslash C$ since it can vanish at two distinct points (if they lie in the same connected component). Actually, this is a distance on the (finite) set of the connected components of $S^{n} \backslash C$. It is well known that the homology class of the intersection of two cycles only depends on the homology class of the cycles. We use this fact in the next proposition to derive a property of the distance $d_{C}$ which will be useful for our purpose.

Proposition 3.2 The value of $d_{C}(\lambda ; \mu) \bmod 2$ is independent of the allowed path joining $\lambda$ and $\mu$. 
Proof Let $\gamma_{1}$ and $\gamma_{2}$ be two allowed paths joining $\lambda$ and $\mu$. The union $\gamma$ of these two allowed paths defines a 1-geometric cycle $\gamma$. Therefore, as $C$ is an Euler cycle, the homology class (in $H_{0}\left(S^{n} ; \mathbb{Z}_{2}\right)$ ) of $C \cap \gamma$ only depends on the homology classes of $C$ and $\gamma$.

But, since $H^{1}\left(S^{n} ; \mathbb{Z}_{2}\right)$ is zero, the Poincare dual of the fundamental class of $C$ is zero, and thus its cup product with the dual of the fundamental class of $\gamma$ vanishes as well. On the other hand, by definition, if [.] denotes the dual of the fundamental class, we have

$$
\lg _{C} \gamma \equiv[\gamma([0 ; 1]) \cap C]
$$

(identifying $H^{n}\left(S^{n} ; \mathbb{Z}_{2}\right)$ with $\left.\mathbb{Z}_{2}\right)$. Hence, $\lg _{C} \gamma_{1}+\lg _{C} \gamma_{2} \equiv 0$, as required.

Note that an immediate consequence of Proposition 3.2 is

$$
d_{C}(\lambda ; \mu) \equiv d_{C}(\lambda ; \tau)+d_{C}(\tau ; \mu)
$$

for any $\lambda, \tau$ and $\mu$ in $S^{n} \backslash C$.

Remark 1 We may associate a graph $G_{C}$ to $C$, whose vertices are the connected components of $S^{n} \backslash C$ and where the edges are connecting two vertices $V$ and $V^{\prime}$ satisfying $\operatorname{dim} c l(V) \cap \operatorname{cl}\left(V^{\prime}\right) \geq n-1$. The distance $d_{C}$ is now the geodesic distance in the graph. Proposition 3.2 says that the graph $G_{C}$ is bi-colorable.

\subsection{Multiplicity $\bmod 2$ and $d_{C}$}

In this section $A$ is the germ of an analytic hypersurface of $\mathbb{R}^{n+1}$. The relation between $d_{C}$ and the multiplicity is given by the following proposition.

Proposition 3.3 For any $\lambda \in S^{n} \backslash C_{A}$, we have

$$
m_{A} \equiv d_{C_{A}^{\prime}}(\lambda ;-\lambda)
$$

Proof Choose a generic fiber of $\pi_{\lambda}^{\prime}$. This provides an allowed path from $\lambda$ to $-\lambda$. Its length modulo 2 is $m_{\lambda}\left(C_{A}^{\prime}\right)$ if the fiber is sufficiently generic. But, by Proposition 2.4, $m_{\lambda}\left(C_{A}^{\prime}\right) \equiv m_{A}$.

As a consequence we see that $d_{C_{A}^{\prime}}(\lambda ;-\lambda)$ only depends on the connected component of $S^{n} \backslash C_{A}$ where $\lambda$ is chosen. The number $m_{\lambda}$ merely says whether $\lambda$ and $-\lambda$ are of the same color in the coloring of $S_{n} \backslash C_{A}^{\prime}$.

Definition 3.4 We will say that a set $C \subseteq S^{n}$ is $a$-invariant if it is preserved globally by the antipodal map (i.e. $a(C)=C$, with $a(x)=-x)$. In this case $d_{C}(-\lambda ;-\mu)=$ $d_{C}(\lambda ; \mu)$ for any $\lambda$ and $\mu$ in $S^{n} \backslash C$.

Proposition 3.5 Modulo 2, the number $d_{C}(\lambda ;-\lambda)$ is independent of $\lambda \in S^{n} \backslash C$ if and only if $C$ is a-invariant. 
Proof To prove the "only if" remark, by (3.1) we have

$$
d_{C}(-\lambda ;-\lambda) \equiv d_{C}(\lambda ; \mu)+d_{C}(\mu ;-\mu)+d_{C}(-\mu ;-\lambda)
$$

Then the result follows from $d_{C}(\lambda ; \mu)=d_{C}(-\mu ;-\lambda)$. For the "if" part, as $C$ is closed, it is enough to show $a(x) \in C$, for $x$ in a dense subset of $C$. Take $x$ generic in $C$, with $a(x)$ not in $C$. If $x$ is sufficiently generic, then $C$ is a topological manifold at $C$. The set $C$ separates a little neighborhood of $x$ in $S^{n}$ in two connected components. Thus, we may pick $\lambda$ and $\mu$ close to $x$ such that $d_{C}(\lambda ; \mu)=1$. Using the above identity, we see that if $d_{C}(\lambda ;-\lambda) \equiv d_{C}(\mu ;-\mu)$ then

$$
d_{C}(-\lambda ;-\mu) \equiv 1
$$

But as $a(x)$ does not belong to $C, d_{C}(\lambda ; \mu)=0$, if $\lambda$ and $\mu$ are chosen sufficiently close to $x$. This is a contradiction.

Remark 2 As a consequence of Propositions 3.3 and 3.5, the odd part of the tangent cone is $a$-invariant. In view of the two above propositions it is natural to set $m_{C}: \equiv$ $d_{C}(\lambda ;-\lambda)$, for a generic $\lambda$, so that now

$$
m_{A}=m_{C_{A}^{\prime}}
$$

It is already enough to establish the invariance of the multiplicity modulo 2 under arc-analytic bi-Lipschitz mappings. Arc-analytic bi-Lipschitz mapping are studied in [4] where it is shown that the inverse of a bi-Lipschitz arc-analytic map is arcanalytic.

Theorem 3.6 Multiplicity mod2 is an arc-analytic bi-Lipschitz invariant for real analytic hypersurfaces of $\mathbb{R}^{n}$.

Proof Let $A$ and $B$ be two germs of analytic sets and let $h$ be an arc-analytic biLipschitz homeomorphism sending $A$ onto $B$. By Proposition 2.5, $h^{\prime}$ is a homeomorphism sending $C_{A}^{\prime}$ onto $C_{B}^{\prime}$. Observe that, as $h$ is arc-analytic, a ray $r \mapsto r \lambda$ (with $\lambda \in S^{n}$ ) is sent onto an analytic arc which may not be a cusp since $h$ is bi-Lipschitz. This clearly means that the tangent half-lines at 0 of the image of this analytic arc have opposite directions, that is to say $h^{\prime}(-\lambda)=-h^{\prime}(\lambda)$. Therefore,

$$
m_{A}=d_{C_{A}^{\prime}}(\lambda ;-\lambda)=d_{C_{B}^{\prime}}\left(h^{\prime}(\lambda) ; h^{\prime}(-\lambda)\right)=m_{B}
$$

It is proved in [6] that the lines $\left\{x^{2}=y^{3}\right\}$ and $\left\{x^{3}=y^{7}\right\}$ are arc-analytically homeomorphic. Hence, the hypothesis "bi-Lipschitz" may not be omitted.

Remark 3 As the Yang index (see [11]) is preserved by equivariant mappings, the Yang index of $C_{A}^{\prime}$ is an arc-analytic bi-Lipschitz invariant. 


\section{On $a$-Invariant Hypersurfaces of $S^{n}$}

\subsection{The Case of $S^{2}$}

In this section we focus on hypersurfaces of $S^{2}$. This will enable us to prove the invariance through a subanalytic bi-Lipschitz homeomorphism of the multiplicity $\bmod 2$ for hypersurfaces of $\mathbb{R}^{3}$ in Sect. 4. Actually, in this setting we have a better result. The multiplicity mod 2 may be characterized just by means of the distance $d_{C}$ introduced above. This fact is no longer true in higher dimensions.

More precisely, let us define the diameter of an Euler cycle $C \subset S^{2}$ as the integer

$$
\delta_{C}=\sup \left\{d_{C}(\lambda ; \mu) / \lambda, \mu \in S^{2} \backslash C\right\} .
$$

Theorem 4.1 If $C$ is an a-invariant Euler cycle of $S^{2}$ of dimension one, then

$$
\delta_{C} \equiv m_{C} .
$$

Proof We need to check that $\delta_{C} \equiv d_{C}(-\lambda ; \lambda)$, for some $\lambda \in S^{2} \backslash C$. Let $\lambda$ and $\mu$ be such that $\delta_{C}=d_{C}(\lambda ; \mu)$. We actually are going to see that

$$
d_{C}(\lambda ; \mu) \leq \max \left(d_{C}(\lambda ;-\lambda) ; d_{C}(\mu ;-\mu)\right) .
$$

Let $\gamma$ be an allowed path from $\lambda$ to $-\lambda$ realizing the distance. Observe that $-\gamma$ is also such a path. The union $\gamma \cup-\gamma$ separates the sphere into at least two connected components. Remark that, as this path is $a$-invariant, the points $\mu$ and $-\mu$ may not lie in the same connected component of $S^{2} \backslash \gamma \cup-\gamma$ (see for instance [5, Corollary 3]). It means that there is a geodesic $\sigma$ from $\mu$ to $-\mu$ which cuts a geodesic $\sigma^{\prime}$ from $\lambda$ to $-\lambda$ (either $\gamma$ or $-\gamma$ ). Let $\theta$ be a connected component crossed by both $\sigma$ and $\sigma^{\prime}$.

Note that, as $\theta$ is crossed by a geodesic from $\mu$ to $-\mu$, we have

$$
d_{C}(\mu ; \theta)+d_{C}(\theta ;-\mu)=d_{C}(\mu ;-\mu)
$$

so that if $d_{C}(\theta ;-\mu) \geq d_{C}(\lambda ; \theta)$ then we get

$$
d_{C}(\lambda ; \mu) \leq d_{C}(-\mu ; \mu) .
$$

Hence, we may suppose $d_{C}(\theta ;-\mu) \leq d_{C}(\lambda ; \theta)$. But then as we also have

$$
d_{C}(\lambda ; \theta)+d_{C}(\theta ;-\lambda)=d_{C}(\lambda ;-\lambda)
$$

we get

$$
d_{C}(-\lambda ;-\mu) \leq d_{C}(\lambda ;-\lambda) .
$$

As $d_{C}(\lambda ; \mu)=d_{C}(-\lambda ;-\mu)$, we are done.

Even for a hypersurface of $S^{3}$, the above proposition is no longer true. For instance, the torus

$$
\left\{x \in \mathbb{R}^{4}: x_{1}^{2}+x_{2}^{2}=\frac{1}{2}, x_{3}^{2}+x_{4}^{2}=\frac{1}{2}\right\}
$$


is an $a$-invariant subset of $S^{3}$ and has a diameter equal to 1 while the distance from a connected component to its opposite is zero.

\subsection{On $a$-Invariant Submanifolds of $S^{n}$}

We will say for short that an $a$-invariant Euler cycle $C$ of $S^{n}$ is even if $m_{C}=0$ and odd if $m_{C}=1$ (see Remark 2). The question raised by Sect. 2 is the following: can an even hypersurface of $S^{n}$ be homeomorphic to an odd one?

We answer negatively in this section in some particular cases. We start by considering the case of manifolds that are submanifolds of an odd dimensional sphere. In this case, it is known that we can have a characterization of the multiplicity in terms of the Euler characteristic (see for instance [10, 1.3.B]).

Proposition 4.2 Let $C$ be an a-invariant subanalytic submanifold of $S^{2 k+1}$ of dimension $2 k$. Then

$$
m_{C} \equiv \frac{1}{2} \chi(C)
$$

Proof Let $\pi: S^{2 k+1} \rightarrow \mathbb{R} P^{2 k+1}$ be the quotient map and let $C^{\prime}:=\pi(C)$. Observe that $m_{C} \equiv 0$ if and only if the number of intersection points of $C^{\prime}$ with a generic projective line is even, which means that the number of intersection points with any closed subanalytic generic closed path is even.

Therefore, if $m_{C}=0$ then we may associate a color (say either white or black) to each connected component of $\mathbb{R} P^{2 k+1} \backslash C^{\prime}$ such that two adjacent connected components have opposite colors. But, due to Poincaré duality, this implies that

$$
\chi\left(C^{\prime}\right)=\sum_{F \in \mathcal{C}} \chi(\partial F)=\sum_{F \in \mathcal{C}} 2 \cdot \chi(F),
$$

where $\mathcal{C}$ is the family constituted by the closure of the white connected components. As $\chi(C)=2 \chi\left(C^{\prime}\right)$ this proves the result in the case where $m_{C}$ is even.

Now, if $m_{C}$ is nonzero, we may stratify $C^{\prime}$ and choose a generic projective hyperplane $P$ cutting the strata transversely so that $P \cap C^{\prime}$ is a topological manifold of dimension $2 k-1$. This implies that

$$
\chi\left(P \cap C^{\prime}\right)=0 .
$$

Observe that $P \cup C^{\prime}$ is even and so, like in the even case, we may choose a coloring of the complement. The same computation yields

$$
\chi\left(P \cup C^{\prime}\right) \equiv 0,
$$

which means that $\chi\left(C^{\prime}\right)$ is odd, from which we conclude that $\frac{1}{2} \chi(C)$ is odd.

Proposition 4.2 does generalize to singular sets, as is shown by Fig. 1.

Figure 1 shows a singular $a$-invariant subset of $\mathbb{R}^{3} \cup\{p t\} \approx S^{3}$. It is invariant with respect to the involution of $S^{3}$ (and not that of $\mathbb{R}^{3}$ ). Thus, the two pinched handles 
Fig. $1 C \subset \mathbb{R}^{3} \approx S^{3} \cup\{\infty\}$

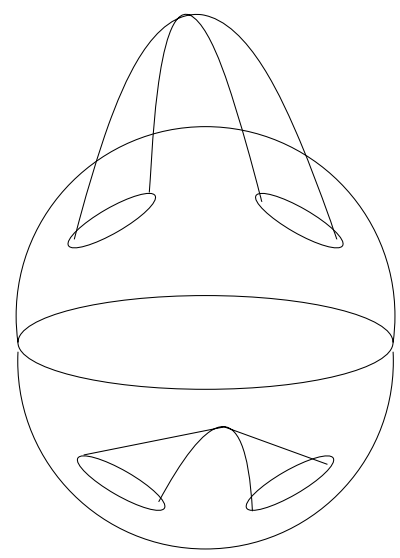

are interchanged by the antipodal map. This set is odd, but the Euler characteristic is zero. However, we are going to show that in the case where we have only isolated singularities, an even subset may not be homeomorphic to an odd one (Proposition 4.3).

In the case where we only have isolated singularities, we may deduce the result invariance of the multiplicity by deleting the singularities.

Proposition 4.3 Let $C_{1}$ and $C_{2}$ be two a-invariant subanalytic hypersurfaces of $S^{2 k+1}$ which have only isolated singularities. Assume that there exists a homeomorphism $h: S^{2 k+1} \rightarrow S^{2 k+1}$ which maps $C_{1}$ onto $C_{2}$. Then $C_{1}$ is even iff $C_{2}$ is.

Proof Assume that $C_{1}$ is odd and $C_{2}$ even. We first choose a coloring of the family of the connected components of the complement of $C_{1}$ and $C_{2}$, as explained in Remark 1 . We may assume that $h$ preserves the colors. Pulling back the antipodal map by $h$ (i.e. setting $\left.* x:=h^{-1}(-h(x))\right)$ we get an involution $*$ on $S^{n}$ preserving $C_{1}$ and preserving the colors.

We denote by $W$ the union of all the white connected components of the complement of $C_{1}$ and by $B$ the union of all the black ones. Let us define

$$
\Lambda_{=}:=\left\{x \in \operatorname{Sing} C_{1}: \chi(W \cap S(x ; \varepsilon))=\chi(B \cap S(x ; \varepsilon))\right\},
$$

where $\operatorname{Sing} C_{1}$ denotes the points at which $C_{1}$ is not a topological manifold. It is well known that $\Lambda_{=}$is independent of $\varepsilon$ if it is chosen small enough. Let also $\Lambda_{\neq}:=$ Sing $C_{1} \backslash \Lambda_{=}$.

Observe that $\Lambda_{=}$and $\Lambda_{\neq}$are $a$-invariant subsets. Moreover, since we have assumed that the singularities are isolated, these sets are finite. We shall define a map $c: \Lambda_{\neq} \rightarrow\{B, W\}$, equivariant for both involutions - and $*$. Actually, it is enough to set

$$
c(\lambda)=W, \quad \text { if } \chi(B \cap S(x ; \varepsilon))<\chi(W \cap S(x ; \varepsilon)) \quad \text { and } \quad c(\lambda)=B \text {, otherwise. }
$$


Choose also a mapping $d: \Lambda_{=} \rightarrow\{W, B\}$ such that $d(-x)=-d(x)$ (it is clearly possible since - has no fixed point). Now, let us set

$$
C_{-}:=\left(C_{1} \backslash \bigcup_{x \in \operatorname{Sing} C_{1}} B(x ; \varepsilon)\right) \cup\left(\bigcup_{x \in \Lambda_{=}} S(x ; \varepsilon) \cap d(x)\right) \cup\left(\bigcup_{x \in \Lambda_{\neq}} S(x ; \varepsilon) \cap c(x)\right) .
$$

It defines an $a$-invariant subset of $S^{2 k+1}$. Define now an equivariant subset of $S^{2 k+1}$ invariant under the action of $*$ by setting

$$
\begin{aligned}
C_{*}:= & \left(C_{2} \backslash \bigcup_{x \in \operatorname{Sing} C_{2}} B(h(x) ; \varepsilon)\right) \cup\left(\bigcup_{x \in \Lambda_{=}} S(h(x) ; \varepsilon) \cap h(W)\right) \\
& \cup\left(\bigcup_{x \in \Lambda_{\neq}} S(h(x) ; \varepsilon) \cap c^{\prime}(x)\right),
\end{aligned}
$$

where $c^{\prime}(x)=h(c(x))$.

Note that, as $C_{1}$ has been modified only locally at the singularities, the values of $d_{C}$ have been modified only for the points lying close to $\operatorname{Sing} C_{1}$ and therefore $C_{-}$is still odd and $C_{*}$ is still even.

Remark that as we have cut off the singular locus, the set $C^{\prime}:=C_{1} \backslash$ $\bigcup_{x \in \operatorname{Sing} C_{1}} B(x ; \varepsilon)$ is a manifold with boundary. Near $x \in \operatorname{Sing} C_{1}$, this boundary is also the boundary of the closure of the manifolds $S(x ; \varepsilon) \cup W$ and $S(x ; \varepsilon) \cup B$. Therefore, near every $x \in \operatorname{Sing} C_{1}$, the sets $C^{\prime} \cup(S(x ; \varepsilon) \cap W)$ and $C^{\prime} \cup(S(x ; \varepsilon) \cap W)$ are topological manifolds and hence so are $C_{-}$and $C_{*}$. We claim that

$$
\chi\left(C_{-}\right)=\chi\left(C_{*}\right)
$$

which will clearly contradict Proposition 4.2 since we have already observed that $C_{-}$ is even and $C_{*}$ odd.

To prove this claim, first remark that the sets $C_{-}$and $C_{*}$ are the respective disjoint unions of three sets. The first and the third set involved in each union are germs and links at singular points of homeomorphic sets. As the Euler characteristic of the link is a topological invariant of the singularity, they have the same Euler characteristic. Thus, it is enough to prove that the Euler characteristics of the second set coincide, i.e.,

$$
\sum_{x \in \Lambda_{=}} \chi(S(h(x) ; \varepsilon) \cap h(W))=\sum_{x \in \Lambda_{=}} \chi(S(x ; \varepsilon) \cap d(x)) .
$$

But, by definition of $\Lambda_{=}$we have

$$
\chi(S(x ; \varepsilon) \cap W)=\chi(S(x ; \varepsilon) \cap B),
$$

for any $x \in \Lambda_{=}$. This implies the preceding equality (no matter whether $d(x)$ is $W$ or $B$ ) and yields the claim.

Remark 4 Actually, the odd hypersurfaces of $S^{n}$ are those having Yang's index equal to $(n-1)$ (see $[5,11]$ ). Hence, we have proved in this section that an $a$-invariant 
subanalytic subset of $S^{n}$ with only isolated singularities cannot be homeomorphic to one having a Yang index strictly inferior.

\section{Multiplicity $\bmod 2$ and Bi-Lipschitz Maps}

\subsection{The Main Results}

We end this article by proving four equimultiplicity theorems that are consequences of the main results of the previous section. We start by the case of hypersurfaces of $\mathbb{R}^{3}$.

Theorem 5.1 Multiplicity mod 2 is a subanalytic bi-Lipschitz invariant for real analytic hypersurfaces of $\mathbb{R}^{3}$.

Proof Let $A$ be the germ of a real analytic hypersurface of $\mathbb{R}^{3}$. By Propositions 3.3 and 2.4 we have

$$
m_{A}=d_{C_{A}^{\prime}}(\lambda ;-\lambda)
$$

for $\lambda \notin C_{A}^{\prime}$. By Theorem 4.1 we know that the number $d_{C_{A}^{\prime}}(\lambda ;-\lambda)$ is a topological invariant of $C_{A}^{\prime}$. But by Proposition 2.5 if an analytic germ $A$ is subanalytically biLipschitz homeomorphic to an analytic germ $B$ then $C_{A}^{\prime}$ is homeomorphic to $C_{B}^{\prime}$.

We also can state the following criteria of equimultiplicity $\bmod 2$.

Theorem 5.2 Let $A$ be the germ of an analytic hypersurface of $\mathbb{R}^{n}$ with $n$ even. Assume that $C_{A}$ has only isolated singularities. Then the multiplicity mod 2 of $A$ is preserved by subanalytic bi-Lipschitz homeomorphisms.

Let us make the statement of the above theorems a little more precise. By "the multiplicity mod 2 of $A$ is preserved by subanalytic bi-Lipschitz homeomorphisms" we mean that another germ of analytic hypersurface $B$ of $\mathbb{R}^{n}$, for which we have a germ of subanalytic bi-Lipschitz homeomorphism $h:\left(\mathbb{R}^{n} ; 0\right) \rightarrow\left(\mathbb{R}^{n} ; 0\right)$ carrying $A$ onto $B$, has the same multiplicity $\bmod 2$ as $A$. Actually the theorem holds not only for analytic hypersurfaces but for all Euler cycles having a "well-defined multiplicity" (that is to say an $a$-invariant odd part of the tangent cone, see Proposition 3.5).

Proof This can be checked in the same way as for Theorem 5.1, replacing Theorem 4.1 by Proposition 4.3. Note that if $C_{A}$ has only isolated singularities, $C_{A}^{\prime}$ has isolated singularities as well.

Remark that Proposition 4.2 shows that when $n$ is even it is not necessary to have a homeomorphism of the ambient space.

In the case where $n$ is odd we may prove the metric invariance of the multiplicity if $C_{A}^{\prime}$ is a manifold. This is due to the fact that we may take the suspension of the odd part of the tangent cone. 
Theorem 5.3 If $C_{A}^{\prime}$ is a topological manifold, the conclusion of the previous theorem holds for any $n$.

Proof We may assume that $n$ is odd. Observe that the suspension of $C_{A}^{\prime}$ provides an $a$-equivariant subset of $S^{n}$, which has only isolated singularities. The respective suspensions of two homeomorphic sets are clearly homeomorphic. Thus, we may apply exactly the same argument as in the previous theorem.

Of course, if $C_{A}$ is a topological manifold then so is $C_{A}^{\prime}$.

In order to state the last equimultiplicity theorem, we introduce a notation. Given a germ of analytic hypersurface $A \subset \mathbb{R}^{n+1}$, let

$$
\rho_{A}:=\sup _{x \in S^{n}} d\left(x ; C_{A}^{\prime}\right)
$$

(where $C_{A}^{\prime}$ is defined by Proposition 2.4).

We are going to see that two germs of real analytic sets $A$ and $B$ which are subanalytically bi-Lipschitz homeomorphic have the same multiplicity when the Lipschitz constant is close enough to one. In the complex case, a similar theorem has been proved by Comte in [2].

In the following theorem, the bound for the Lipschitz constant of $h$ could be slightly improved but this complicates the computations. Actually, the most important fact is that we give a bound which only depends on $A$.

Theorem 5.4 Let $A$ and $B$ be germs at 0 of real analytic hypersurfaces of $\mathbb{R}^{n+1}$. Let $h:\left(\mathbb{R}^{n+1} ; 0\right) \rightarrow\left(\mathbb{R}^{n+1} ; 0\right)$ be the germ of a subanalytic bi-Lipschitz homeomorphism with $|\operatorname{Lip}(h)-1| \leq \frac{\rho_{A}^{2}}{48}$ and $h(A)=B$. Then $m_{A} \equiv m_{B}$.

Proof By Proposition 2.5, the mapping $h^{\prime}: S^{n} \rightarrow S^{n}$ satisfies $h^{\prime}\left(C_{A}^{\prime}\right)=C_{B}^{\prime}$ and, by Lemma 2.6, (2.4) holds. Set for simplicity $L:=\operatorname{Lip}(h)$ and $\delta:=L-1$.

Take $x \in S^{n}$ such that $d\left(x ; C_{A}^{\prime}\right)=\rho_{A}$. First we observe that, by definition of $\rho_{A}$, we have

$$
d\left(h^{\prime}(x) ; C_{B}^{\prime}\right) \geq \frac{\rho_{A}}{C^{2}}-\frac{\rho_{A}^{2}}{48} \geq \rho_{A}-2 \frac{\rho_{A}^{2}}{48} \rho_{A}-\frac{\rho_{A}^{2}}{48} \geq \frac{3}{4} \rho_{A}
$$

(since $\frac{1}{L^{2}} \geq 1-2 \delta$ and $\rho_{A} \leq 2$ ).

Now remark that if

$$
d\left(-h^{\prime}(-x) ; h^{\prime}(x)\right)<d\left(h^{\prime}(x) ; C_{B}^{\prime}\right),
$$

then $-h^{\prime}(-x)$ and $h^{\prime}(x)$ belong to the same connected component of $S^{n} \backslash C_{B}^{\prime}$, and hence, by Propositions 3.3 and 3.2, the conclusion of the theorem ensues. Therefore, thanks to the previous inequality, it is enough to show that

$$
d\left(-h^{\prime}(-x) ; h^{\prime}(x)\right)<\frac{3}{4} \rho_{A} .
$$


But by (2.4):

$$
d\left(h^{\prime}(x) ; h^{\prime}(-x)\right) \geq \frac{2}{L^{2}}-\frac{\rho_{A}^{2}}{48} \geq 2-5 \frac{\rho_{A}^{2}}{48} .
$$

By Pythagoras this implies that

$$
d\left(h^{\prime}(x) ;-h^{\prime}(-x)\right) \leq \sqrt{\frac{20 \rho_{A}^{2}}{48}}<\frac{3}{4} \rho_{A},
$$

as required.

\subsection{A Conjecture}

The general case still remains undecided, even in $\mathbb{R}^{4}$. As the results of Sects. 1 and 2 are general, it is enough to generalize Proposition 4.3. In other words, the subanalytic bi-Lipschitz invariance of the multiplicity $\bmod 2$ for real analytic hypersurfaces would be established if the following conjecture were proved.

Conjecture Let $C$ and $C^{\prime}$ be two $a$-invariant Euler cycles of $S^{n}$ of dimension $(n-1)$. Assume that there exists a subanalytic homeomorphism $h: S^{n} \rightarrow S^{n}$ such that $h(C)=C^{\prime}$. Then $C$ is even iff so is $C^{\prime}$ (see Sect. 3 for the definition).

It seems to be true at least in $S^{3}$ (on pictures). Note that it is enough to solve the problem for odd dimensional spheres (or even dimensional) since we may take the suspension of the subsets.

Acknowledgements This paper was begun when the author was invited by the Jagiellonian University of Kraków. Part of this paper was completed when the author was visiting the Department of Mathematics at the University of Provence. The author is grateful to Anna Valette for her careful reading of the manuscript and to Jan Jaworowski for valuable discussions. The author also thanks the referees for their useful remarks.

\section{References}

1. Bochnak, J., Coste, M., Roy, M.-F.: Géométrie Algébrique Réelle. Ergebnisse der Math., vol. 12. Springer, Berlin (1987)

2. Comte, G.: Multiplicity of complex analytic sets and bi-Lipschitz maps. In: Real Analytic and Algebraic Singularities, Nagoya/Sapporo/Hachioji, 1996. Pitman Res. Notes Math. Ser., vol. 381, pp. 182 188. Longman, Harlow (1998). 32S05 (32S50)

3. Ferrarotti, M., Fortuna, E., Wilson, L.: Real algebraic varieties with prescribed tangent cones. Pac. J. Math. 194(2), 315-323 (2000)

4. Fukui, T., Kurdyka, K., Paunescu, L.: An inverse mapping theorem for arc-analytic homeomorphisms. In: Geometric Singularity Theory. Banach Center Publ., vol. 65, pp. 49-56. Polish Acad. Sci., Warsaw (2004)

5. Jaworowski, J.W.: On antipodal sets on the sphere and on continuous involutions. Fund. Math. 43, 241-254 (1956)

6. Kobayashi, M., Kuo, T.-C.: Uniqueness of unibranched curve in $\mathbb{R}^{2}$ up to simple blowings up. Proc. Jpn. Acad. Ser. A Math. Sci. 73(6), 97-99 (1997)

7. Kurdyka, K.: Ensembles semi-algébriques symétriques par arcs. Math. Ann. 282(3), 445-462 (1988) 
8. Kurdyka, K., Raby, G.: Densité des ensembles sous-analytiques. Ann. Inst. Fourier (Grenoble) 39(3), 753-771 (1989)

9. Valette, G.: Lipschitz triangulations. Ill. J. Math. 49(3), 953-979 (2005)

10. Viro, O.: Mutual position of hypersurfaces in projective space. Am. Math. Soc. Transl. (2) 186, 161176 (1998)

11. Yang, C.-T.: On theorems of Borsuk-Ulam, Kakutani-Yamabe-Yujobô and Dyson. II. Ann. Math. 62(2), 271-283 (1955) 